\title{
Invariant Measures Associated to Degenerate Elliptic Operators
}

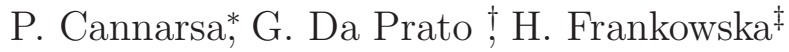

August 6, 2009

\begin{abstract}
This paper is devoted to the study of the existence and uniqueness of the invariant measure associated to the transition semigroup of a diffusion process in a bounded open subset of $\mathbb{R}^{n}$. For this purpose, we investigate first the invariance of a bounded open domain with piecewise smooth boundary showing that such a property holds true under the same conditions that insure the invariance of the closure of the domain. A uniqueness result for the invariant measure is obtained in the class of all probability measures that are absolutely continuous with respect to Lebesgue's measure. A sufficient condition for the existence of such a measure is also provided.
\end{abstract}

Key words: stochastic differential equation, invariance, degenerate elliptic operator, invariant measure.

MSC Subject classifications: 60H10, 47D07, 35K65, 37L40.

\section{Introduction}

Given a compact set $K \subset \mathbb{R}^{n}$, let us consider the transition semigroup

$$
P_{t} \varphi(x) \doteq \mathbb{E}[\varphi(X(t, x))] \quad x \in K, \varphi \in C(K)
$$

\footnotetext{
*Dipartimento di Matematica, Università di Roma "Tor Vergata", Via della Ricerca Scientifica 1, 00133 Roma (Italy), e-mail: cannarsa@mat.uniroma2.it

${ }^{\dagger}$ Scuola Normale Superiore di Pisa, Piazza dei Cavalieri 7, I-56125 Pisa (Italy), e-mail: daprato@sns.it

${ }^{\ddagger}$ CNRS, C \& O, Université Pierre et Marie Curie (Paris 6), case 189, 4 place Jussieu, 75252 Paris (France), e-mail: frankowska@math.jussieu.fr
} 
corresponding to the diffusion process $X(t, x)$ associated with the stochastic differential equation

$$
\left\{\begin{array}{l}
d X(t)=b(X(t)) d t+\sigma(X(t)) d W(t) \quad t \geq 0 \\
X(0)=x
\end{array}\right.
$$

Naturally, in order for $P_{t}$ to be well-defined, a preliminary problem to address is the invariance of $K$ under the stochastic flow of (1.1). On the other hand, even when $K$ is invariant, the infinitesimal generator of $P_{t}$ may be difficult to identify for diffusions in general space dimension $n \geq 2$. However, the complete analysis of the one-dimensional case by Feller [20] shows that no extra boundary conditions are necessary when the diffusion never reaches the boundary of $K$. For this reason, we are also interested in conditions ensuring the invariance of the open domain ${ }^{K}$.

The invariance of a closed domain $K$ under a given diffusion has been investigated by several authors. First, for $K$ of class $C^{3}$ and sufficiently smooth coefficients $b, \sigma$, Friedman [19] studied the invariance of $K$ using the distance function and the elliptic operator

$$
L_{0} \varphi(x)=\frac{1}{2} \operatorname{Tr}\left[a(x) \nabla^{2} \varphi(x)\right]+\langle b(x), \nabla \varphi(x)\rangle,
$$

where $a(x)=\sigma(x) \sigma^{*}(x)$. In particular, in [19] it is shown that, if $\partial K$ is regular, then a sufficient condition for the invariance of $K$ is

$$
\left\{\begin{array}{ll}
(i) & L_{0} \delta_{K}(x) \geq 0 \\
(i i) & \left\langle a(x) \nabla \delta_{K}(x), \nabla \delta_{K}(x)\right\rangle=0
\end{array} \quad \forall x \in \partial K,\right.
$$

where $\delta_{K}$ stands for the oriented distance from $\partial K$. Notice that condition (ii) implies that $a(x)$ is a singular matrix for all $x \in \partial K$.

Following this, in [1], Aubin and the second author introduced the notion of stochastic contingent cone to provide necessary and sufficient conditions for the viability of $K$ - as well as its invariance - under minimal regularity assumptions. Another approach to the problem was proposed in [7], using viscosity solutions of a second order Hamilton-Jacobi equation. Later on, in $[5,6]$, second order jets were used to study invariance and viability, while in [12] the second and third author applied the Stratonovich drift to give first order necessary and sufficient conditions for the invariance of an arbitrary closed set for a stochastic control system. Then, using the distance function, in $[10,14]$ a condition similar to $(1.2)$ was shown to be necessary and sufficient for the invariance of closed convex sets, while in [11] a sufficient condition for the invariance of the interior was derived. 
In this paper, we begin the analysis considering the invariance problem for an open set $\stackrel{\circ}{K}$ with piecewise smooth boundary. Such a problem was studied by Friedman and Pinsky [17] (see also [18, chapter 9]) for $C^{3}$-smooth domains and coefficients $b, \sigma$ of class $C^{1}$ : they proved that (1.2) is a sufficient condition for the invariance of $\stackrel{\circ}{K}$. In section 3 , we will further investigate the above problem showing that condition (1.2) is indeed necessary and sufficient for the invariance of the interior of $K$ under milder regularity assumptions, see Theorem 3.2.

Then, using the invariance of the interior of $K$, we study the transition semigroup showing, first, that its infinitesimal generator on $C(K)$ is given by operator $L_{0}$ above. Consequently, for every $\lambda>0$ and every continuous function $f: K \rightarrow \mathbb{R}$, we obtain an existence and uniqueness result for the elliptic equation

$$
\lambda \varphi-L_{0} \varphi=f \quad \text { in } \quad K
$$

without imposing boundary conditions.

Finally, we apply our results to study the existence and uniqueness of invariant measures for $P_{t}$. Observe that, since $K$ is bounded, $P_{t}$ always admits at least one invariant measure. On the other hand, unlike the semigroups that are associated with operators defined in the whole space $\mathbb{R}^{n}$ (see e.g. [23] and the references therein), $P_{t}$ can have several invariant measures, which, moreover, need not be absolutely continuous with respect to Lebesgue's measure. In this paper, taking advantage of the interior invariance result described above, we prove that $P_{t}$ has at most one invariant measure on $C(K)$, in the class of all probability measures that are absolutely continuous with respect to Lebesgue's measure. Moreover, strengthening condition (1.2), we are able to prove the existence of such a measure.

This paper is organized as follows: section 2 contains notations and all preliminary results; section 3 develops our interior invariance result for piecewise smooth domains. Section 4 provides the characterization of the infinitesimal generator of $P_{t}$. Finally, section 5 is devoted to the analysis of the invariant measure for $P_{t}$, absolutely continuous with respect to Lebesgue's measure. We conclude with a few examples and an appendix.

\section{Notation}

Given a metric space $(\mathcal{E}, d), \mathcal{B}(\mathcal{E})$ stands for the Borel $\sigma$-algebra in $\mathcal{E}$, and $B_{b}(\mathcal{E})$ for the space of all bounded Borel functions $\varphi: \mathcal{E} \rightarrow \mathbb{R}$.

Let $n$ be a positive integer. We denote by:

- $\langle\cdot, \cdot\rangle$ the Euclidean scalar product in $\mathbb{R}^{n}$; 
- $|\cdot|$ the Euclidean norm in $\mathbb{R}^{n}$;

- $e_{j}=(\overbrace{0, \ldots, 0}^{j-1}, 1,0, \ldots, 0)$, where $j=1, \ldots, n$, the elements of the canonical base of $\mathbb{R}^{n}$;

- $x \otimes y$ the tensor product of $x, y \in \mathbb{R}^{n}$, i.e., $(x \otimes y) z=\langle y, z\rangle x$ for all $z \in \mathbb{R}^{n}$

- $B\left(x_{0}, r\right)$ the open ball of radius $r>0$, centered at $x_{0} \in \mathbb{R}^{n}$, and we set $B_{r}=B(0, r)$;

- $\mathcal{L}\left(\mathbb{R}^{n}, \mathbb{R}^{m}\right)$ the space of all linear maps $\Lambda: \mathbb{R}^{n} \rightarrow \mathbb{R}^{m}$, where $m$ is a positive integer, and any element $\sigma \in \mathcal{L}\left(\mathbb{R}^{n}, R^{m}\right)$ will be identified with the unique $n \times m$ matrix that represents $\sigma$ with respect to the canonical bases of $\mathbb{R}^{n}$ and $\mathbb{R}^{m}$;

- $\|\Lambda\|$ the operator norm of $\Lambda \in \mathcal{L}\left(\mathbb{R}^{n}, \mathbb{R}^{m}\right)$, i.e., $\|\Lambda\|=\max _{|x|=1}|\Lambda x|$;

- $\operatorname{Tr}[\Lambda]$ the trace of $\Lambda \in \mathcal{L}\left(\mathbb{R}^{n}, \mathbb{R}^{n}\right)$, i.e., $\operatorname{Tr}[\Lambda]=\sum_{j}\left\langle\Lambda e_{j}, e_{j}\right\rangle$;

- $\mu_{n}$ the Lebesgue measure on $\mathcal{B}\left(\mathbb{R}^{n}\right)$;

- $\mathbb{1}_{S}$ the characteristic function of a set $S$;

- $\nabla \varphi, \nabla^{2} \varphi$ and $\Delta \varphi$ the gradient vector, the Hessian matrix, and the Laplacian of the function $\varphi$, respectively.

Given a positive integer $m$ and Lipschitz continuous maps $b: \mathbb{R}^{n} \rightarrow \mathbb{R}^{n}$ and $\sigma: \mathbb{R}^{n} \rightarrow \mathcal{L}\left(\mathbb{R}^{n} ; \mathbb{R}^{m}\right)$, consider the stochastic differential equation

$$
\left\{\begin{array}{l}
d X(t)=b(X(t)) d t+\sigma(X(t)) d W(t) \quad t \geq 0 \\
X(0)=x
\end{array}\right.
$$

where $W(t)$ is a standard $m$-dimensional Brownian motion on a complete filtered probability space $\left(\Omega, \mathcal{F},\left\{\mathcal{F}_{t}\right\}_{t \geq 0}, \mathbb{P}\right)$. It is well-known that, for any $x \in \mathbb{R}^{n}$, problem (2.1) has a unique solution that we shall denote by $X(\cdot, x)$. Moreover, $X(\cdot, x)$ is $\mathbb{P}-$ a.s. continuous.

Let $S \subset \mathbb{R}^{n}$ be a nonempty set. We denote by $d_{S}$ the Euclidean distance function from $S$, that is,

$$
d_{S}(x)=\inf _{y \in S}|x-y| \quad \forall x \in \mathbb{R}^{n} .
$$


It is well-known that $d_{S}$ is a Lipschitz function of constant 1. If $S$ is closed, then the above infimum is a minimum, which is attained on a set that will be called the projection of $x \in \mathbb{R}^{n}$ onto $S$, labeled $\operatorname{proj}_{S}(x)$, that is,

$$
\operatorname{proj}_{S}(x)=\left\{y \in S|| x-y \mid=d_{S}(x)\right\} \quad \forall x \in \mathbb{R}^{n} .
$$

We say that $S$ is invariant for $X(\cdot, \cdot)$ iff

$$
x \in S \quad \Longrightarrow \quad X(t, x) \in S \quad \mathbb{P} \text { - a.s. } \quad \forall t \geq 0 .
$$

For every $x \in S$, the hitting time of $\partial S$ is the random variable defined by

$$
\tau_{S}(x)=\inf \{t \geq 0: X(t, x) \in \partial S\} .
$$

Let $K$ be a closed subset of $\mathbb{R}^{n}$ with nonempty interior $\stackrel{\circ}{K}$ and boundary $\partial K$. A well-known function in metric analysis is the so-called oriented distance from $\partial K$, that is, the function

$$
\delta_{K}(x)= \begin{cases}d_{\partial K}(x) & \text { if } x \in K \\ -d_{\partial K}(x) & \text { if } x \in K^{c}\end{cases}
$$

In what follows we will use the following sets, defined for any $\varepsilon>0$ :

- $\mathcal{N}_{\epsilon}=\left\{x \in \mathbb{R}^{n}:\left|\delta_{K}(x)\right|<\epsilon\right\}$

- $K_{\varepsilon}=K \cap \mathcal{N}_{\varepsilon}$

- $\stackrel{\circ}{K}_{\varepsilon}=\stackrel{\circ}{K} \cap \mathcal{N}_{\varepsilon}$

In this paper, we will use the following function spaces:

$C_{b}(A)$ : all bounded continuous functions on the open set $A$;

$C^{2,1}(A)$ : all twice differentiable functions on $A$, with bounded Lipschitz second order derivatives;

$C_{\text {loc }}^{2,1}(A)$ : all twice differentiable functions on $A$, with locally Lipschitz second order derivatives;

$C(K)$ : all continuous functions $\varphi: K \rightarrow \mathbb{R} ;$

$C^{1}(K)$ : all continuously differentiable functions in a neighborhood of $K$;

$H^{2}(A)$ : the Sobolev space of all Borel functions $\varphi: A \rightarrow \mathbb{R}$ that are square integrable on $A$, together with their second order derivatives in the sense of distributions. 
$H_{\text {loc }}^{2}(A)$ : all Borel functions $\varphi: A \rightarrow \mathbb{R}$ that belong to $H^{2}\left(A^{\prime}\right)$ for every open set $A^{\prime}$ such that $\overline{A^{\prime}} \subset A$.

We say that $K$ is a closed domain of class $C^{2,1}$ if it is a closed connected subset of $\mathbb{R}^{n}$ such that for all $x \in \partial K$ there exist $r>0$ and a function $\phi: B(x, r) \rightarrow \mathbb{R}$ of class $C^{2,1}(B(x, r))$ such that

$$
\partial K \cap B(x, r)=\{y \in B(x, r) \mid \phi(y)=0\} .
$$

More generally, we shall say that $K$ is a piecewise $C^{2,1}$-smooth domain if

$$
K=\bigcap_{j=1}^{m} K^{j}
$$

where $K^{j}$ are closed domains of class $C^{2,1}$. It is well-known that

$$
K \text { compact domain of class } C^{2,1} \Longleftrightarrow \exists \varepsilon_{0}>0: \delta_{K} \in C^{2,1}\left(\mathcal{N}_{\varepsilon_{0}}\right)
$$

see, e.g., [16]. A useful consequence of the above property is that

$$
\forall x \in K_{\varepsilon_{0}} \quad \begin{cases}(a) & \exists ! \bar{x} \in \partial K: \delta_{K}(x)=|x-\bar{x}| \\ (b) & \nabla \delta_{K}(x)=\nabla \delta_{K}(\bar{x})=-\nu_{K}(\bar{x})\end{cases}
$$

where $\nu_{K}(\bar{x})$ stands for the outward unit normal to $K$ at $\bar{x}$.

It is easy to see that, if $K$ is a compact domain of class $C^{2,1}$, then there is a sequence $\left\{Q_{i}\right\}$ of compact domains of class $C^{2,1}$ such that

$$
Q_{i} \subset \stackrel{\circ}{Q}_{i+1} \quad \text { and } \quad \bigcup_{i=1}^{\infty} Q_{i}=\stackrel{\circ}{K} .
$$

Indeed, owing to (2.3), it suffices to take, for all $i$ large enough,

$$
Q_{i}=\left\{x \in \mathbb{R}^{n} \mid \delta_{K}(x) \geq \frac{1}{i}\right\} .
$$

Finally, we observe that, if $K$ is a compact set and $\left\{Q_{i}\right\}$ is an increasing sequence of compact domains of class $C^{2,1}$ satisfying (2.5), then $\forall x \in \stackrel{\circ}{K}$

$$
\left\{\begin{array}{l}
\exists i_{x} \in \mathbb{N}: \tau_{Q_{i}}(x)<\tau_{Q_{i+1}}(x)<\tau_{K}(x), \quad \forall i \geq i_{x} \quad \mathbb{P}-\text { a.s. } \\
\lim _{i \rightarrow \infty} \tau_{Q_{i}}(x)=\tau_{K}(x)
\end{array}\right.
$$

Indeed, let $x \in \stackrel{\circ}{K}$ and let $i_{x}$ be the first integer $i$ such that $x \in Q_{i}$. Then, since $X(\cdot, x)$ is continuous, $\left\{\tau_{Q_{i}}(x)\right\}_{i \geq i_{x}}$ is an increasing sequence of random 
variables bounded above by $\tau_{K}(x)$. So, $\left\{\tau_{Q_{i}}(x)\right\}$ converges to some random variable $\tau(x)$ which satisfies $\tau(x) \leq \tau_{K}(x)$. If $\mathbb{P}\left(\tau(x)<\tau_{K}(x)\right)>0$, then

$$
\mathbb{P}\left(\tau(x)<t_{0}<\tau_{K}(x)\right)>0
$$

for some $t_{0}>0$. Consequently,

$$
X\left(t_{0}, x\right) \notin \cup_{i} Q_{i} \quad \mathbb{P}-\text { a.s. } \quad \text { on } \quad\left\{\tau(x)<t_{0}<\tau_{K}(x)\right\} .
$$

So,

$$
X\left(t_{0}, x\right) \in \partial K \quad \mathbb{P}-\text { a.s. } \quad \text { on } \quad\left\{\tau(x)<t_{0}<\tau_{K}(x)\right\},
$$

in contrast with the definition of $\tau_{K}(x)$.

\section{Invariance of the interior of $K$}

In this section, we will study the invariance properties of a compact piecewise $C^{2,1}$-smooth domain $K$ with respect to the flow $X(\cdot, \cdot)$ associated with equation (2.1) (with Lipschitz continuous coefficients $b$ and $\sigma$ ).

Necessary and sufficient conditions for the invariance of the compact set $K$ were formulated in [10] in terms of the differential operator

$$
\left\{\begin{array}{l}
D\left(L_{0}\right)=\left\{\varphi \in C(K) \mid \varphi \in H_{\mathrm{loc}}^{2}(\stackrel{\circ}{K}), L_{0} \varphi \in C(K)\right\} \\
L_{0} \varphi(x):=\frac{1}{2} \operatorname{Tr}\left[a(x) \nabla^{2} \varphi(x)\right]+\langle b(x), \nabla \varphi(x)\rangle \quad x \in K,
\end{array}\right.
$$

where $a(x)$ is defined in terms of the diffusion coefficient $\sigma$ :

$$
a(x)=\sigma(x) \sigma^{*}(x) \geq 0 \quad \forall x \in K
$$

From [14] it follows that, if in addition $K$ is convex, then $K$ is invariant with respect to the $X(\cdot, \cdot)$ if and only if the following conditions are satisfied:

$$
\left\{\begin{array}{ll}
(i) & L_{0} \delta_{K}(x) \geq 0 \\
(i i) & \left\langle a(x) \nabla \delta_{K}(x), \nabla \delta_{K}(x)\right\rangle=0
\end{array} \quad \forall x \in \partial K\right.
$$

Notice that, on account of (2.4), the above conditions imply that the elliptic operator $L_{0}$ is necessarily degenerate on $\partial K$, in the normal direction to $\partial K$.

We observe that, when $K$ is a smooth domain of class $C^{3}$, condition (3.2) is sufficient for the invariance of the interior $\stackrel{\circ}{K}$ of $K$ in the sense that

$$
\mathbb{P}\left(\tau_{K}(x)<\infty\right)=0 \quad \forall x \in \stackrel{\circ}{K} .
$$


(see [17] and also [18]). Such a property heavily relies on the Lipschitz continuity of $b$ and $\sigma$, as well as on the smoothness of $\partial K$. It is not true, in general, if $b$ and $\sigma$ are just continuous.

We will now generalize and improve the above result assuming that

$$
K=\bigcap_{j=1}^{m} K^{j}
$$

where $K^{j}$ are closed domains of class $C^{2,1}$ with the following property: for some $\varepsilon_{1}>0$ and all $j \in\{1, \ldots, m\}$

$$
\operatorname{proj}_{\partial K^{j}}(x) \in \partial K \quad \forall x \in \stackrel{\circ}{K} \cap K_{\varepsilon_{1}}^{j},
$$

(recall that $K_{\varepsilon_{1}}^{j}=\left\{x \in K^{j}:\left|\delta_{K^{j}}(x)\right|<\varepsilon_{1}\right\}$ ).

For every $x \in \partial K$ we denote by $J(x)$ the set of all active indeces at $x$ :

$$
j \in J(x) \quad \Longleftrightarrow \quad x \in \partial K^{j} .
$$

Using, for simplicity, the abbreviated notation $\delta_{j}$ for the oriented distance

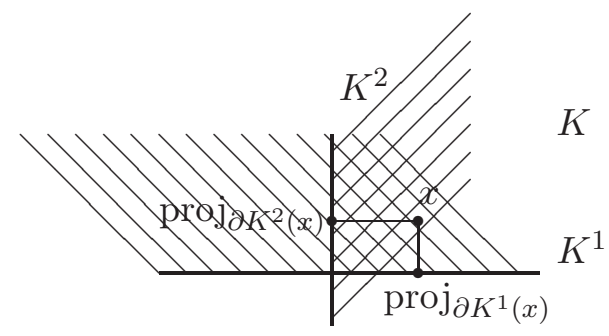

Figure 1: Assumption (3.5).

$\delta_{K^{j}}$, let us also assume that

$$
0 \notin \operatorname{co}\left\{\nabla \delta_{j}(x) \mid j \in J(x)\right\} \quad \forall x \in \partial K .
$$

Then, Clarke's tangent cone to $K$ at every $x \in K$ has nonempty interior. For this reason, $K$ coincides with the closure of $\stackrel{\circ}{K}$. Moreover, according to [4, chapter 4], Clarke's normal cone to $K$ at any point $x \in \partial K$ is given by

$$
N_{K}(x)=\sum_{j \in J(x)} \mathbb{R}_{-} \nabla \delta_{j}(x) .
$$

Finally, we observe that the existence of a sequence $\left\{Q_{i}\right\}$ of compact domains of class $C^{2,1}$ satisfying (2.5) is also guaranteed when $K$ is a compact set with the above properties (3.4), (3.5) and (3.6). 
Example 3.1 A typical example of a piecewise smooth domain satisfying conditions (3.4), (3.5), and (3.6) is the cube

$$
K=\left\{x \in \mathbb{R}^{n}\left|\max _{1 \leq j \leq n}\right| x_{j} \mid \leq 1\right\} .
$$

Notice that $\partial K=\left\{x \in \mathbb{R}^{n}\left|\max _{j}\right| x_{j} \mid=1\right\}$,

$$
J(x)=\left\{j \in\{1, \ldots, n\}|| x_{j} \mid=1\right\} \quad \forall x \in \partial K
$$

and

$$
\nabla \delta_{j}(x)=-\frac{x_{j}}{\left|x_{j}\right|} e_{j} \quad \forall x \in \partial K, \forall j \in J(x) .
$$

We now give our interior invariance result for piecewise smooth domains.

Theorem 3.2 Assume (3.4), (3.5), and (3.6). Then the following three statements are equivalent:

(a) $K$ is invariant;

(b) for all $x \in \partial K$ and $j \in J(x) \begin{cases}(i) & L_{0} \delta_{j}(x) \geq 0 \\ (i i) & \left\langle a(x) \nabla \delta_{j}(x), \nabla \delta_{j}(x)\right\rangle=0 ;\end{cases}$

(c) $\stackrel{\circ}{K}$ is invariant.

Proof: It is not restrictive to assume that $\varepsilon_{1}>0$ is such that, for every $j \in\{1, \ldots, m\}$, there exist functions $g_{j} \in C^{2,1}\left(\mathbb{R}^{n}\right)$ satisfying

$$
\left\{\begin{array}{lll}
0 \leq g_{j} \leq 1 & \text { on } & K^{j} \\
0<g_{j} & \text { on } & K^{j} \backslash K_{\varepsilon_{1}}^{j} \\
g_{j} \equiv \delta_{j} & \text { on } & K_{\varepsilon_{1}}^{j}
\end{array}\right.
$$

Assume (a). Then, according to [12],

$$
\sigma^{*}(x) p=0 \quad \forall x \in \partial K, \forall p \in N_{K}(x) .
$$

Consequently, owing to (3.7), property $(b)(i i)$ holds true. To obtain $(i)$, fix $x \in \partial K$ and let $j \in J(x)$. Then, $g_{j}(X(t, x)) \geq 0 \mathbb{P}-$ a.s. for all $t \geq 0$, and $g_{j}(x)=0$. Therefore,

$$
\frac{d}{d t} \mathbb{E}\left[g_{j}(X(\cdot, x))\right]_{\mid t=0} \geq 0
$$


Therefore, applying Itô's formula (see, e.g., [9, p. 61]),

$$
\frac{d}{d t} \mathbb{E}\left[g_{j}(X(\cdot, x))\right]_{\mid t=0}=\mathbb{E}\left[L_{0} g_{j}(x)\right] \geq 0
$$

Since $g_{j} \equiv \delta_{j}$ on a neighborhood of $\partial K^{j}$, we deduce $(i)$.

We shall now prove that $(b) \Rightarrow(c)$. Let us consider the function

$$
V(x) \doteq-\sum_{j=1}^{m} \log g_{j}(x) \quad \forall x \in \stackrel{\circ}{K} .
$$

Then,

$$
L_{0} V(x)=-\sum_{j=1}^{m} \frac{1}{g_{j}(x)} L_{0} g_{j}(x)+\sum_{j=1}^{m} \frac{1}{g_{j}^{2}(x)}\left|\sigma^{*}(x) \nabla g_{j}(x)\right|^{2} .
$$

We claim that, for all $j=1, \ldots, m$,

$$
\frac{1}{g_{j}^{2}(x)}\left|\sigma^{*}(x) \nabla g_{j}(x)\right|^{2}-\frac{1}{g_{j}(x)} L_{0} g_{j}(x) \leq c \quad \forall x \in \stackrel{\circ}{K}
$$

for some constant $c \geq 0$. Indeed, the above estimate holds true when $\delta_{j}(x) \geq$ $\varepsilon_{1}$ since $g_{j}$ is strictly positive on $\left\{x \in \stackrel{\circ}{K} \mid \delta_{j}(x) \geq \varepsilon_{1}\right\}$. So, we have to prove (3.11) for all $x \in \stackrel{\circ}{K} \cap K_{\varepsilon_{1}}^{j}$, that is,

$$
\frac{1}{\delta_{j}^{2}(x)}\left|\sigma^{*}(x) \nabla \delta_{j}(x)\right|^{2}-\frac{1}{\delta_{j}(x)} L_{0} \delta_{j}(x) \leq c \quad \forall x \in \stackrel{\circ}{K} \cap K_{\varepsilon_{1}}^{j} .
$$

For $x \in \stackrel{\circ}{K} \cap K_{\varepsilon_{1}}^{j}$ let $\bar{x}$ denote the projection of $x$ on the boundary of $K^{j}$. Then, owing to $(i i)$, for all $x \in \stackrel{\circ}{K} \cap K_{\varepsilon_{1}}^{j}$ we have that

$$
\begin{aligned}
\left|\sigma^{*}(x) \nabla \delta_{j}(x)\right| & =\left|\left(\sigma^{*}(x)-\sigma^{*}(\bar{x})\right) \nabla \delta_{j}(x)+\sigma^{*}(\bar{x}) \nabla \delta_{j}(\bar{x})\right| \\
& =\left|\left(\sigma^{*}(x)-\sigma^{*}(\bar{x})\right) \nabla \delta_{j}(x)\right| \\
& \leq\left\|\sigma^{*}(x)-\sigma^{*}(\bar{x})\right\| \\
& \leq c_{1}|x-\bar{x}|=c_{1} \delta_{j}(x)
\end{aligned}
$$

where $c_{1}$ is a Lipschitz constant for $\sigma$. Consequently,

$$
\frac{1}{\delta_{j}^{2}(x)}\left|\sigma^{*}(x) \nabla \delta_{j}(x)\right|^{2} \leq c_{1}^{2} \quad \forall x \in \stackrel{\circ}{K} \cap K_{\varepsilon_{1}}^{j} .
$$

Also, observe that the function

$$
L_{0} \delta_{j}(x)=\frac{1}{2} \operatorname{Tr}\left[\sigma(x) \sigma^{*}(x) \nabla^{2} \delta_{j}(x)\right]+\left\langle b(x), \nabla \delta_{j}(x)\right\rangle
$$


is Lipschitz continuous in $\stackrel{\circ}{K} \cap K_{\varepsilon_{1}}^{j}$. Thus, $(i)$ yields

$$
\begin{aligned}
-\frac{1}{\delta_{j}(x)} L_{0} \delta_{j}(x) & =-\frac{1}{\delta_{j}(x)}\left(L_{0} \delta_{j}(x)-L_{0} \delta_{j}(\bar{x})\right)-\frac{1}{\delta_{j}(x)} L_{0} \delta_{j}(\bar{x}) \\
& \leq \frac{1}{\delta_{j}(x)}\left|L_{0} \delta_{j}(x)-L_{0} \delta_{j}(\bar{x})\right| \\
& \leq \frac{c_{2}}{\delta_{j}(x)}|x-\bar{x}|=c_{2}
\end{aligned}
$$

for all $x \in \stackrel{\circ}{K} \cap K_{\varepsilon_{1}}^{j}$, where $c_{2}$ is a Lipschitz constant for $L_{0} \delta_{j}$. So, combining (3.13) and (3.14), we deduce (3.12).

Now, by (3.11) and (3.10),

$$
L_{0} V(x) \leq M \quad \forall x \in \stackrel{\circ}{K}
$$

for some constant $M \geq 0$. Let us set

$$
V(x)=\lim _{K \ni y \rightarrow x} V(y)=\infty \quad \forall x \in \partial K .
$$

Next, let $\left\{Q_{i}\right\}$ be a sequence of compact domains of class $C^{2,1}$ satisfying (2.5) and consider their stopping times $\tau_{Q_{i}}$. By Itô's formula we have, for all $x \in Q_{i}$ and $t \geq 0$,

$$
\begin{aligned}
V\left(X\left(t \wedge \tau_{Q_{i}}(x), x\right)\right)=V(x) & +\int_{0}^{t \wedge \tau_{Q_{i}}(x)}\left(L_{0} V\right)(X(s, x)) d s \\
& +\int_{0}^{t \wedge \tau_{Q_{i}}(x)}\langle\nabla V(X(s, x)), \sigma(X(s, x)) d W(s)\rangle .
\end{aligned}
$$

Hence, taking expectation and recalling (3.15),

$$
\begin{aligned}
\mathbb{E}\left[V\left(X\left(t \wedge \tau_{Q_{i}}(x), x\right)\right)\right]=V(x)+\mathbb{E} \int_{0}^{t \wedge \tau_{Q_{i}}(x)} & L_{0} V(X(s, x)) d s \\
& \leq V(x)+M t
\end{aligned}
$$

Owing to (2.6) and Fatou's lemma, the above inequality yields

$$
\mathbb{E}\left[V\left(X\left(t \wedge \tau_{K}(x), x\right)\right)\right] \leq V(x)+M t \quad \forall t \geq 0, \forall x \in \stackrel{\circ}{K} .
$$

Since the function in the right-hand above is finite on $\stackrel{\circ}{K}$, we deduce that

$$
\mathbb{P}\left(\tau_{K}(x) \leq t\right)=\mathbb{P}\left(V\left(X\left(t \wedge \tau_{K}(x), x\right)\right)=\infty\right)=0 \quad \forall t \geq 0, \forall x \in \stackrel{\circ}{K}
$$


To conclude that $\stackrel{\circ}{K}$ is invariant, take a sequence $t_{k} \uparrow \infty$ and observe that

$$
0=\mathbb{P}\left(\tau_{K}(x) \leq t_{k}\right) \uparrow \mathbb{P}\left(\tau_{K}(x)<\infty\right) \quad \forall x \in \stackrel{\circ}{K} .
$$

Finally, let us show that $(c)$ implies $(a)$. Suppose $\stackrel{\circ}{K}$ is invariant and fix $x \in K$. Recalling that $K$ coincides with the closure of $\stackrel{\circ}{K}$, let $\left\{x_{k}\right\}$ be a sequence in $\stackrel{\circ}{K}$ such that $x_{k} \rightarrow x$. Then, by our invariance assumption, $X\left(t, x_{k}\right) \in K, \mathbb{P}-$ a.s. for all $t \geq 0$. Since $X\left(t, x_{k}\right) \rightarrow X(t, x), \mathbb{P}-$ a.s. for all $t \geq 0$, we conclude that $X(t, x) \in K, \mathbb{P}-$ a.s., for all $t \geq 0$. Since $x$ is an arbitrary point in $K$, we have shown that $K$ is invariant.

Under a stronger assumption, one can improve the estimates of the above proof to obtain the following result that will be used in section 5.2.

Proposition 3.3 Assume (3.4), (3.5), and (3.6), and suppose

$$
\forall \bar{x} \in \partial K, \forall j \in J(\bar{x}) \quad \begin{cases}(i) & \limsup _{K \ni x \rightarrow \bar{x}} \frac{L_{0} \delta_{j}(x)}{\delta_{j}(x) \log \delta_{j}(x)}<0 \\ (i i) & \left\langle a(\bar{x}) \nabla \delta_{j}(\bar{x}), \nabla \delta_{j}(\bar{x})\right\rangle=0\end{cases}
$$

Then, there is a nonnegative function $V \in C_{\mathrm{loc}}^{2,1}(\stackrel{\circ}{K})$ such that

$$
\begin{cases}V(x)=-\sum_{j=1}^{m} \log \delta_{j}(x) & \forall x \in \stackrel{\circ}{K}_{\varepsilon_{1} / 2} \\ L_{0} V(x) \leq M-\alpha V(x) & \forall x \in \stackrel{\circ}{K}\end{cases}
$$

for some constants $\alpha>0$ and $M \geq 0$.

Remark 3.4 In particular, assumption $(i)$ holds true if

$$
L_{0} \delta_{j}(x)>0 \quad \forall x \in \partial K \cap \partial K^{j}, \quad j=1, \ldots, m .
$$

Observe that the above condition was used in [11] to prove the invariance of $\stackrel{\circ}{K}$ for diffusion processes with a continuous drift.

We sketch the proof of Proposition 3.3 below, focussing on the only point in which it differs from the proof of Theorem 3.2.

Proof: The reasoning goes in the same way as above up to (3.13). Then, in view of assumption $(i)$, there exist positive numbers $\alpha$ and $\rho$ such that

$$
-\frac{1}{\delta_{j}(x)} L_{0} \delta_{j}(x) \leq \alpha \log \delta_{j}(x) \quad \forall x \in \stackrel{\circ}{K} \cap K_{\rho}^{j} .
$$

So, combining (3.10), (3.13) and (3.18), we conclude that, for some $M>0$,

$$
L_{0} V(x) \leq M-\alpha V(x) \quad \forall x \in \stackrel{\circ}{K} .
$$




\section{Transition semigroup}

In this section we will assume the following without further notice:

- $K$ is a compact set satisfying (3.4), (3.5) and (3.6);

- condition (b) of Theorem 3.2 holds true.

We recall that one can find then a sequence $\left\{Q_{i}\right\}$ of compact domains of class $C^{2,1}$ satisfying (2.5), that is $Q_{i} \subset \stackrel{\circ}{Q}_{i+1}$ and $\bigcup_{i=1}^{\infty} Q_{i}=\stackrel{\circ}{K}$.

Then, we know that $K$ and $\stackrel{\circ}{K}$ are invariant for the stochastic flow $X$. So, as recalled above, the elliptic operator $L_{0}$ defined in (3.1) is degenerate on $\partial K$ in the sense specified by condition $(b)$. Later on, we will further assume that $L_{0}$ is uniformly elliptic on all compact subsets of $K$, that is,

$$
\operatorname{det} a(x)>0 \quad \forall x \in \stackrel{\circ}{K} .
$$

The main objective of our analysis is the study of the transition semigroup $P_{t}$ associated with the stochastic flow $X(\cdot, \cdot)$, that is, the semigroup on $B_{b}(K)$ defined by

$$
P_{t} \varphi(x):=\mathbb{E}[\varphi(X(t, x))] \quad \forall \varphi \in B_{b}(K), \forall x \in K, \forall t \geq 0 .
$$

As is easily seen, $P_{t}$ is a Markov semigroup, that is,

$$
\begin{cases}(i) & \varphi \geq \psi \quad \Longrightarrow \quad P_{t} \varphi \geq P_{t} \psi \\ (i i) \quad P_{t} \mathbb{1}_{K}=\mathbb{1}_{K}\end{cases}
$$

We begin with some preliminary properties of $P_{t}$.

Proposition $4.1 P_{t}$ is a Feller semigroup on $B_{b}(K)$, and its restriction to $C(K)$ is strongly continuous.

Proof: The Feller property of $P_{t}$ is easy to check. Indeed,

$$
\varphi \in C(K) \quad \Longrightarrow \quad P_{t} \varphi \in C(K) \quad \forall t \geq 0
$$

owing to the continuity of the map $x \mapsto X(t, x)$. Notice that we will use the same symbol $P_{t}$ to denote the restriction of $P_{t}$ to $C(K)$.

In order to prove that $P_{t}$ is a strongly continuous semigroup on $C(K)$, observe that, since $C^{1}(K)$ is dense in $C(K)$ and $\left\|P_{t}\right\| \leq 1^{(1)}$, it is enough to show that

$$
\lim _{t \downarrow 0} P_{t} \varphi=\varphi \quad \text { uniformly in } \quad K
$$

\footnotetext{
${ }^{(1)}$ Here, $\left\|P_{t}\right\|$ denotes the norm $P_{t}$ regarded as a bounded linear operator on $C(K)$.
} 
for every $\varphi \in C^{1}(K)$. Now, for any such function $\varphi$ we have that

$$
\left|P_{t} \varphi(x)-\varphi(x)\right| \leq\|\varphi\|_{C^{1}(K)}\left\{\mathbb{E}\left[|X(t, x)-x|^{2}\right]\right\}^{1 / 2} \quad \forall x \in K, \forall t \geq 0 .
$$

Moreover, by Hölder's inequality,

$$
\begin{aligned}
|X(t, x)-x|^{2} & \leq 2\left|\int_{0}^{t} b(X(s, x)) d s\right|^{2}+2\left|\int_{0}^{t} \sigma(X(s, x)) d W(s)\right|^{2} \\
& \leq 2 t \int_{0}^{t}|b(X(s, x))|^{2} d s+2\left|\int_{0}^{t} \sigma(X(s, x)) d W(s)\right|^{2} .
\end{aligned}
$$

So, taking expectation yields

$$
\mathbb{E}\left(|X(t, x)-x|^{2}\right) \leq 2 t^{2}\|b\|^{2}+2 t\|\sigma\|^{2}
$$

where we have set $\|b\|=\max _{x \in K}|b(x)|$ and $\|\sigma\|=\max _{x \in K}\|\sigma(x)\|$. Thus, (4.3) follows recalling (4.4).

Remark 4.2 As a corollary of Theorem 3.2, we have that the transition semigroup $P_{t}$ defined in (4.2) satisfies

$$
P_{t} \varphi(x)=\mathbb{E}\left[\varphi(X(t, x)) \mathbb{1}_{t \leq \tau_{K}(x)}\right] \quad \forall t \geq 0, \forall x \in \stackrel{\circ}{K}
$$

for every $\varphi \in B_{b}(K)$. Now, for all $i \in \mathbb{N}$ consider the so-called stopped semigroups

$$
P_{t}^{i} \varphi(x)=\mathbb{E}\left[\varphi(X(t, x)) \mathbb{1}_{t \leq \tau_{Q_{i}}(x)}\right] \quad\left(t \geq 0, x \in Q_{i}\right)
$$

associated with stopping times $\tau_{Q_{i}}(x)$. Then, by (4.5) and (2.6), we conclude that $P_{t}^{i}$ approximate $P_{t}$ on $\stackrel{\circ}{K}$ in the sense that, for every $\varphi \in B_{b}(K)$,

$$
\lim _{i \rightarrow \infty} P_{t}^{i} \varphi(x)=P_{t} \varphi(x) \quad \forall t \geq 0, \forall x \in \stackrel{\circ}{K} .
$$

Remark 4.3 Under hypothesis (4.1) we have that $L_{0}$ is uniformly elliptic on $Q_{i}$ for all $i \in \mathbb{N}$. So, by classical results (see, e.g., [22]), for any $\varphi \in C(K)$ the Dirichlet problem

$$
\begin{cases}\partial_{t} u(t, x)=L_{0} u(t, x) & t \geq 0, x \in Q_{i} \\ u(t, x)=0 & t>0, x \in \partial Q_{i} \\ u(0, x)=\varphi(x) & x \in Q_{i} .\end{cases}
$$


has a unique solution $u_{i} \in C\left([0, \infty) ; L^{p}\left(Q_{i}\right)\right)$ for every $p \geq 1$, which satisfies

$$
\partial_{t} u_{i}(t, \cdot), \partial_{h} \partial_{k} u_{i}(t, \cdot) \in L^{p}\left(Q_{i}\right) \quad \forall t>0, \forall h, k=1, \ldots, n
$$

Moreover, $u_{i}$ is given by the formula

$$
u_{i}(t, x)=P_{t}^{i} \varphi(x) \quad\left(t \geq 0, x \in Q_{i}\right),
$$

where $P_{t}^{i}$ is the semigroup defined in (4.6), see, e.g., [8, section 6.2.2]. Observe, however, that $u_{i}$ can be also represented by the formula

$$
u_{i}(t, x)=\int_{Q_{i}} G_{i}(t, x, y) \varphi(y) d y \quad\left(t \geq 0, x \in Q_{i}\right),
$$

where $G_{i}(t, x, y)$ is the Green function of the parabolic operator in (4.8). It is well-known that $G_{i}(t, x, y)$ is strictly positive for all $t \geq 0$ and $x, y \in \stackrel{\circ}{Q}_{i}$ (see, e.g., [22]). By the maximum principle we conclude that

$$
G_{i}(t, x, y) \uparrow G(t, x, y) \quad \forall t \geq 0, \forall x, y \in \stackrel{\circ}{K}, x \neq y
$$

Therefore,

$$
G(t, x, y)>0 \quad(t \geq 0, x, y \in \stackrel{\circ}{K}, x \neq y) .
$$

Also, on account of $(4.10),(4.11)$ and $(4.7)$, for all $\varphi \in C(K)$ we have

$$
P_{t} \varphi(x)=\int_{K} G(t, x, y) \varphi(y) d y \quad \forall t \geq 0, \forall x \in \stackrel{\circ}{K} .
$$

Let $L$ be the infinitesimal generator of the strongly continuous semigroup $P_{t}$ on $C(K)$. The following theorem ensures that $L$ coincides with operator $L_{0}$.

Theorem 4.4 Assume (4.1) and let $\lambda>0$. Then, for every $f \in C(K)$ there exists a unique solution $\varphi^{f} \in D\left(L_{0}\right)$ of the equation

$$
\lambda \varphi-L_{0} \varphi=f \quad \text { in } K .
$$

Moreover, $\varphi^{f} \in D(L)$ and $L \varphi^{f}=L_{0} \varphi^{f}$.

The above result can be proved in several ways. In particular, it can be obtained in a more general framework using viscosity solutions. For the sake of completeness, in the appendix we provide a self-contained proof of Theorem 4.4 which only requires classical tools. 


\section{Invariant measure for $P_{t}$}

In this section, we will study the existence and uniqueness of the invariant measure $\mu$ for the transition semigroup $P_{t}$ defined by (4.2), in the class of all absolutely continuous measures with respect to Lebesgue's measure $\mu_{n}$. We will make, without further notice, the following assumptions:

- $K$ is a compact set satisfying (3.4), (3.5) and (3.6);

- condition (b) of Theorem 3.2 holds true;

- the interior ellipticity condition (4.1) is satisfied.

Let $\left\{Q_{i}\right\}$ be a sequence of compact domains of class $C^{2,1}$ satisfying (2.5).

Let us recall that a probability measure $\mu$ on $(K, \mathcal{B}(K))$ is said to be invariant for $P_{t}$ if, for any $t \geq 0$,

$$
\int_{K} P_{t} \varphi(x) \mu(d x)=\int_{K} \varphi(x) \mu(d x) \quad \forall \varphi \in C(K) .
$$

\section{$5.1 \quad$ Uniqueness}

We will show the following uniqueness result.

Theorem 5.1 $P_{t}$ possesses at most one invariant measure in the class of all probability measures that are absolutely continuous with respect to $\mu_{n}$.

For the proof of the above theorem we will need several intermediate steps. To begin, let us introduce the following metric $\rho_{K}$ in $\stackrel{\circ}{K}$ :

$$
\rho_{K}(x, y)=\left|\frac{1}{\delta_{K}(x)}-\frac{1}{\delta_{K}(y)}\right|+|x-y|, \quad x, y \in \stackrel{\circ}{K} .
$$

It is easy to see that $\left(\stackrel{\circ}{K}, \rho_{K}\right)$ is a complete metric space.

Remark 5.2 It is worth noting that a set $Q \subset \stackrel{\circ}{K}$ is compact in $\left(\stackrel{\circ}{K}, \rho_{K}\right)$ if and only $Q$ is compact in $\mathbb{R}^{n}$ with the Euclidean metric. Indeed, suppose that $Q$ is compact in $\mathbb{R}^{n}$. Then, $Q \subset Q_{i}$ for some positive integer $i$. Thus, for all $x \in Q, \delta_{K}(x)>0$. Consider any sequence $x_{k} \in Q$ and $x \in Q$ such that $\left|x_{k}-x\right| \rightarrow 0$. Then, $\rho_{K}\left(x, x_{k}\right) \rightarrow 0$. Consequently, $Q$ is also compact in $\left(\stackrel{\circ}{K}, \rho_{K}\right)$. Conversely, assume that $Q$ is compact in $\left(\stackrel{\circ}{K}, \rho_{K}\right)$ and let $x, x_{k} \in Q$ be such that $\rho_{K}\left(x, x_{k}\right) \rightarrow 0$. Then, $\left|x_{k}-x\right| \rightarrow 0$. So, $Q$ is compact in the Euclidean metric. 
Taken $\left(\stackrel{\circ}{K}, \rho_{K}\right)$, consider the semigroup

$$
\stackrel{\circ}{P}_{t} \varphi(x):=\mathbb{E}[\varphi(X(t, x))] \quad \forall \varphi \in B_{b}(\stackrel{\circ}{K}), x \in \stackrel{\circ}{K}, t \geq 0 .
$$

Recall that a probability measure $\mu$ on $(\stackrel{\circ}{K}, \mathcal{B}(\stackrel{\circ}{K}))$ is invariant for $\stackrel{\circ}{P}_{t}$ iff

$$
\int_{\check{K}} \stackrel{\circ}{P}_{t} \varphi(x) \mu(d x)=\int_{\check{K}} \varphi(x) \mu(d x) \quad \forall \varphi \in C_{b}(\stackrel{\circ}{K}), t \geq 0 .
$$

Our next result is intended to compare the notion of invariant measure for $P_{t}$ with the one for $\stackrel{\circ}{P}_{t}$.

Lemma 5.3 (a) If $\mu<<\mu_{n}$ is an invariant measure for $P_{t}$, then its restriction to $(\stackrel{\circ}{K}, \mathcal{B}(\stackrel{\circ}{K}))$ is an invariant measure for $\stackrel{\circ}{P}_{t}$.

(b) If $\mu<<\mu_{n}$ is an invariant measure for $\stackrel{\circ}{P}_{t}$, then it can be uniquely extended to an invariant measure for $P_{t}$.

Proof. First of all, we observe that, in view of definitions (4.2) and (5.3),

$$
P_{t} \varphi(x)=\stackrel{\circ}{P}_{t} \varphi(x) \quad \forall t \geq 0, \forall x \in \stackrel{\circ}{K},
$$

where $\varphi$ denotes any function in $C(K)$ as well as its restriction to $\stackrel{\circ}{K}$.

(a) Let $\mu<<\mu_{n}$ be an invariant measure for $P_{t}$, and let $\varphi \in C_{b}\left(\AA_{K}^{\circ}\right)$. We shall localize $\phi$ in a neighborhood of each domain $Q_{i}$ : take the positive sequence

$$
\varepsilon_{i}=\min _{x \in Q_{i}} \delta_{K}(x) \quad \forall i \geq 1
$$

and define

$$
\varphi_{i}(x) \doteq \begin{cases}\varphi(x)\left[1-\frac{1}{\varepsilon_{i}} d_{Q_{i}}(x)\right]_{+} & \text {if } x \in \stackrel{\circ}{K} \\ 0 & \text { if } x \in \partial K\end{cases}
$$

where $[s]_{+}=\max \{s, 0\}$. Then, $\varphi_{i} \in C(K)$. Moreover,

$$
\left|\varphi_{i}(x)\right| \leq|\varphi(x)| \quad \text { and } \quad \lim _{i \rightarrow \infty} \varphi_{i}(x)=\varphi(x) \quad \forall x \in \stackrel{\circ}{K}
$$

because $\varphi \equiv \varphi_{i}$ on $Q_{i}$. Therefore,

$$
\lim _{i} \int_{K} \varphi_{i}(x) \mu(d x)=\int_{K} \varphi(x) \mu(d x)=\int_{K} \varphi(x) \mu(d x),
$$


where the last equality can be justified recalling that $\mu<<\mu_{n}$ and observing that, since $\partial K$ is piecewise smooth, $\mu_{n}(\partial K)=0$. Also, owing to (5.6) and definition (5.3), we obtain

$$
\left|\stackrel{\circ}{P}_{t} \varphi_{i}(x)\right| \leq \sup _{\mathscr{K}}|\varphi| \quad \text { and } \quad \lim _{i} \stackrel{\circ}{P}_{t} \varphi_{i}(x)=\stackrel{\circ}{P}_{t} \varphi(x) \quad \forall x \in \stackrel{\circ}{K} .
$$

So, recalling (5.5), by the Dominated Convergence theorem we obtain

$$
\int_{K} P_{t} \varphi_{i}(x) \mu(d x)=\int_{\dot{K}} \stackrel{\circ}{P}_{t} \varphi_{i}(x) \mu(d x) \stackrel{i \rightarrow \infty}{\longrightarrow} \int_{\check{K}^{\circ}} \stackrel{\circ}{P}_{t} \varphi(x) \mu(d x) .
$$

On account of (5.7) and (5.8) $\mu$ is invariant for $\stackrel{\circ}{P}_{t}$.

(b) Let $\mu$ be an invariant measure for $\stackrel{\circ}{P}_{t}$, absolutely continuous with respect to Lebesgue's measure, and let $\varphi \in C(K)$. Then $\varphi$ restricted to $\stackrel{\circ}{K}$ is a bounded continuous function. Thus, again by $(5.5)$,

$$
\begin{aligned}
& \int_{K} \varphi(x) \mu(d x)=\int_{\mathscr{K}} \varphi(x) \mu(d x)=\int_{\mathscr{K}} \stackrel{\circ}{P}_{t} \varphi(x) \mu(d x) \\
&=\int_{\mathscr{K}} P_{t} \varphi(x) \mu(d x)=\int_{K} P_{t} \varphi(x) \mu(d x) .
\end{aligned}
$$

So, $\mu$ is invariant for $P_{t}$.

Our next result establishes important properties of $\stackrel{\circ}{P}_{t}$.

Lemma 5.4 The transition semigroup $\stackrel{\circ}{P}_{t}$ is irreducible and strongly Feller.

Proof: Let us first prove that $\stackrel{\circ}{P}_{t}$ is irreducible, that is, for every open subset $A$ of $\left(\stackrel{\circ}{K}, \rho_{K}\right)$,

$$
\stackrel{\circ}{P}_{t} \mathbb{1}_{A}(x)>0 \quad \forall t>0, \forall x \in \stackrel{\circ}{K} .
$$

Let $x_{0} \in A$ and let $B\left(x_{0}, r\right)$ be contained in $A$ together with its closure. Then, $B\left(x_{0}, r\right) \subset Q_{i}$ for some integer $i$. So, recalling (4.11), by the maximum principle we obtain

$$
\stackrel{\circ}{P}_{t} \mathbb{1}_{B\left(x_{0}, r\right)}(x) \geq P_{t}^{i} \mathbb{1}_{B\left(x_{0}, r\right)}(x)=\int_{Q_{i}} G_{i}(t, x, y) d y>0 .
$$

So, $\stackrel{\circ}{P}_{t}$ is irreducible.

Let us now show that $\stackrel{\circ}{P}_{t}$ is strongly Feller, that is,

$$
\stackrel{\circ}{P}_{t} \varphi \in C_{b}(\stackrel{\circ}{K}) \quad \forall t>0, \forall \varphi \in B_{b}(\stackrel{\circ}{K}) .
$$


For any $\varphi \in B_{b}(\stackrel{\circ}{K})$, all $t>0$ and all positive integers $i$, we know that $P_{t}^{i} \varphi_{\mid Q_{i}} \in C\left(Q_{i}\right)$ since the stopped semigroup $P_{t}^{i}$ is strongly Feller by wellknown regularity properties of solutions to parabolic equations. On the other hand, for any compact set $Q$ in $\left(\stackrel{\circ}{K}, \rho_{K}\right)$ or, equivalently (according to Remark 5.2), in $K$, we have that

$$
\begin{aligned}
\left|P_{t} \varphi(x)-P_{t}^{i} \varphi(x)\right| & \leq \mathbb{E}\left[| \varphi ( X ( t , x ) ) | \left(1-\mathbb{1}_{\left.\left.t \leq \tau_{Q_{i}}(x)\right)\right]}\right.\right. \\
& \leq \sup _{\dot{K}}|\varphi| \mathbb{P}\left(\tau_{Q_{i}}(x)<t\right) \quad \forall x \in Q .
\end{aligned}
$$

Now, in view of Theorem 3.2, property (2.6) ensures that, for any $t \in(0, \infty)$,

$$
\mathbb{P}\left(\tau_{Q_{i}}(x)<t\right) \downarrow 0 \quad(i \rightarrow \infty) \quad \forall x \in \stackrel{\circ}{K} .
$$

So, Dini's Theorem implies that the above convergence is uniform on $Q$, which yields, in turn, the continuity of $\stackrel{\circ}{P}_{t} \varphi$ on $\stackrel{\circ}{K}$.

Proof of Theorem 5.1: Let $\mu$ and $\widetilde{\mu}$ be two invariant measures ${ }^{(2)}$ for $P_{t}$, both absolutely continuous with respect to Lebesgue's measure. Then, in view of Lemma $5.3(a)$, their restrictions to $(\stackrel{\circ}{K}, \mathcal{B}(\stackrel{\circ}{K}))$ - still labeled $\mu$ and $\widetilde{\mu}$ - are invariant for $\stackrel{\circ}{P}_{t}$. Therefore, Khas'minskii's regularity result (see, e.g., [15, Proposition 4.1.1]) and Doob's uniqueness theorem (see, e.g., [15, Theorem 4.2.1]) ensure that $\mu$ and $\widetilde{\mu}$ coincide on $(\stackrel{\circ}{K}, \mathcal{B}(\stackrel{\circ}{K}))$. So, they coincide on $K$ as well, since $\mu, \widetilde{\mu}<<\mu_{n}$.

We conclude this section with two useful properties of $\stackrel{\circ}{P}_{t}$.

Proposition 5.5 Let $\mu$ be an invariant measure for $\stackrel{\circ}{P}_{t}$. Then

(a) $\mu<<\mu_{n}$;

(b) for any $\varphi \in C_{b}(\stackrel{\circ}{K})$

$$
\lim _{t \rightarrow \infty} \stackrel{\circ}{P}_{t} \varphi(x)=\int_{K} \varphi(y) \mu(d y) \quad \forall x \in \stackrel{\circ}{K} .
$$

Proof: Let $\mu$ be an invariant measure for $\stackrel{\circ}{P}_{t}$, and let $B \in \mathcal{B}(\stackrel{\circ}{K})$ be such that $\mu_{n}(B)=0$. Since $\mu$ is a regular measure, from (5.4) we deduce, by a standard approximation argument, that

$$
\int_{\stackrel{\circ}{K}} \stackrel{\circ}{P}_{t} \mathbb{1}_{B}(x) \mu(d x)=\int_{\stackrel{\circ}{K}} \mathbb{1}_{B}(x) \mu(d x)=\mu(B) .
$$

\footnotetext{
${ }^{(2)}$ In particular, both $\mu$ and $\widetilde{\mu}$ are probability measures.
} 
Then, owing to (4.13),

$$
\mu(B)=\int_{\tilde{K}}\left(\int_{B} G(t, x, y) d x\right) \mu(d x),
$$

where $G$ is Green's function. Since $\int_{B} G(t, x, y) d x=0,(a)$ follows.

Finally, property $(b)$ is an immediate consequence of Doob's theorem (see, e.g., [15, Theorem 4.2.1]).

\subsection{A sufficient condition for existence}

In this section we will give sufficient conditions for the existence of an invariant measure $\mu$ for $P_{t}$, absolutely continuous with respect to $\mu_{n}$.

Let us recall that a family $\left\{\mu_{t}\right\}_{t \geq 0}$ of probability measures on a complete metric space $\mathcal{E}$ is said to be tight if, for any $\varepsilon>0$, there exists a compact subset $Q_{\varepsilon}$ of $\mathcal{E}$ such that $\mu_{t}\left(Q_{\varepsilon}\right) \geq 1-\varepsilon$ for every $t \geq 0$.

Now, denote by $\pi_{t}(x, \cdot)$ the law of $X(t, x)$, that is, the measure

$$
\pi_{t}(x, A)=\mathbb{P}(X(t, x) \in A) \quad \forall A \in \mathcal{B}(K) .
$$

Lemma 5.6 Let $x_{0} \in \stackrel{\circ}{K}$ be such that

$$
\mathbb{E}\left[\sum_{j=1}^{m}\left|\log \delta_{j}\left(X\left(t, x_{0}\right)\right)\right|\right] \leq C \quad \forall t \geq 0
$$

for some $C \geq 0$. Then $\left\{\pi_{t}\left(x_{0}, d y\right)\right\}_{t \geq 0}$ is tight.

Proof: For any integer $i$, let $Q_{i}^{c}=\stackrel{\circ}{K} \backslash Q_{i}$ and consider the positive sequence

$$
\varepsilon_{i}=\min _{x \in Q_{i}} \delta_{K}(x) .
$$

Since $\delta_{K}(x)<\varepsilon_{i}$ on $Q_{i}^{c}$, we have

$$
\begin{aligned}
\pi_{t}\left(x_{0}, Q_{i}^{c}\right)=\int_{Q_{i}^{c}} \pi_{t}\left(x_{0}, d y\right) & \leq \frac{1}{\left|\log \varepsilon_{i}\right|} \int_{K} \sum_{j=1}^{m}\left|\log \delta_{j}(y)\right| \pi_{t}\left(x_{0}, d y\right) \\
& =\frac{1}{\left|\log \varepsilon_{i}\right|} \mathbb{E}\left[\sum_{j=1}^{m}\left|\log \delta_{j}\left(X\left(t, x_{0}\right)\right)\right|\right] \leq \frac{C}{\left|\log \varepsilon_{i}\right|} .
\end{aligned}
$$

Since $\varepsilon_{i} \rightarrow 0$ as $i \rightarrow \infty$, the above inequality implies that, given $\epsilon>0$,

$$
\pi_{t}\left(x_{0}, Q_{i}\right)=1-\pi_{t}\left(x_{0}, Q_{i}^{c}\right)>1-\epsilon \quad \forall t \geq 0,
$$

for all $i$ large enough. So, $\left\{\pi_{t}\left(x_{0}, d y\right)\right\}_{t \geq 0}$ is tight.

Our next result completes the analysis of the existence and uniqueness of the invariant measure for $P_{t}$. 
Theorem 5.7 Assume

$$
\forall \bar{x} \in \partial K, \forall j \in J(\bar{x}) \quad \begin{cases}(i) & \limsup _{K \ni x \rightarrow \bar{x}} \frac{L_{0} \delta_{j}(x)}{\delta_{j}(x) \log \delta_{j}(x)}<0 \\ (i i) & \left\langle a(\bar{x}) \nabla \delta_{j}(\bar{x}), \nabla \delta_{j}(\bar{x})\right\rangle=0\end{cases}
$$

Then $P_{t}$ possesses a unique invariant measure $\mu<<\mu_{n}$.

Proof: Since uniqueness is granted by Theorem 5.1, let us concentrate on existence. Suppose we can find an invariant measure for the semigroup $\stackrel{\circ}{P}_{t}$ that we introduced in (5.3). Then, $\mu$ would be absolutely continuous with respect to $\mu_{n}$ in view of Proposition $5.5(a)$. Thus, on account of Lemma $5.3(b), \mu$ would also be extendable to an invariant measure for $P_{t}$, which would obviously remain absolutely continuous with respect to $\mu_{n}$. So, to complete the proof it is enough to construct an invariant measure for $\stackrel{\circ}{P}_{t}$.

Now, the Krylov-Bogoliubov theorem (see, e.g. [9, Theorem 7.1]) ensures that $\stackrel{\circ}{P}_{t}$ possesses an invariant measure if, for some $x_{0} \in \stackrel{\circ}{K}$, the family of probability measures $\left\{\pi_{t}\left(x_{0}, d y\right)\right\}_{t \geq 0}$ is tight. So, thanks to Lemma 5.6, it suffices to obtain (5.10). Let $\alpha>0$ and $V$ be given by Proposition 3.3. Fix $x_{0} \in \stackrel{\circ}{K}$, apply Itô's formula to $V\left(X\left(t, x_{0}\right)\right)$, and take expectation to obtain

$$
\mathbb{E}\left[V\left(X\left(t, x_{0}\right)\right)\right]=V\left(x_{0}\right)+\mathbb{E} \int_{0}^{t}\left(L_{0} V\right)\left(X\left(s, x_{0}\right)\right) d s \quad \forall t \geq 0 .
$$

Then, taking into account (3.17),

$$
\frac{d}{d t} \mathbb{E}\left[V\left(X\left(t, x_{0}\right)\right)\right]=\mathbb{E}\left[\left(L_{0} V\right)\left(X\left(t, x_{0}\right)\right)\right] \leq M-\alpha \mathbb{E}\left[V\left(X\left(t, x_{0}\right)\right)\right] .
$$

This yields

$$
\mathbb{E}\left[V\left(X\left(t, x_{0}\right)\right)\right] \leq e^{-\alpha t} V\left(x_{0}\right)+\frac{M}{\alpha} \quad \forall t \geq 0 .
$$

Since $V$ coincides with $\sum_{j=1}^{m}\left|\log \delta_{j}\left(X\left(t, x_{0}\right)\right)\right|$ near $\partial K,(5.10)$ follows.

\subsection{Examples}

We conclude with three examples describing possible applications of our invariance result.

Example 5.8 Let us consider the stochastic differential equation (2.1) in the closed unit ball $K=\bar{B}_{1} \subset \mathbb{R}^{2}$, where $b: \bar{B}_{1} \rightarrow \mathbb{R}^{2}$ is a Lipschitz vector field and $\sigma$ is defined as follows. Let

$$
\nu(x)=\frac{\left(x_{1}, x_{2}\right)}{|x|}, \quad \xi(x)=\frac{\left(x_{2},-x_{1}\right)}{|x|} \quad \forall x \in \bar{B}_{1} \backslash B_{2 / 3},
$$


and let $\theta \in C^{1}([0,1])$ be such that

$$
0 \leq \theta \leq 1, \quad \theta \equiv\left\{\begin{array}{lll}
1 & \text { on } & B_{1 / 3} \\
0 & \text { on } & \bar{B}_{1} \backslash B_{2 / 3}
\end{array}\right.
$$

Define, for every $x \in \bar{B}_{1}$,

$$
\sigma(x)=\theta(|x|) I+(1-\theta(x))\left[\left(1-|x|^{2}\right) \nu(x) \otimes \nu(x)+\lambda \xi(x) \otimes \xi(x)\right]
$$

where $I$ is the identity matrix and $\lambda \in \mathbb{R}$. Then, it is easy to check that

$$
L_{0} \delta_{K}(x)=-\frac{\lambda^{2}}{2}-\langle b(x), x\rangle \quad \forall x \in \partial K .
$$

Therefore, by Theorem 3.2 we have that $B_{1}$ is invariant for $X$ if and only if

$$
\max _{|x|=1}\langle b(x), x\rangle \leq-\frac{\lambda^{2}}{2} .
$$

Moreover, owing to Theorem 5.1, semigroup $P_{t}$ has at most one invariant measure $\mu<<\mu_{2}$. Furthermore, such a measure does exist if

$$
\max _{|x|=1}\langle b(x), x\rangle<-\frac{\lambda^{2}}{2} .
$$

Example 5.9 In the closed cube $Q_{1} \subset \mathbb{R}^{n}$ (see Example 3.1) let us consider the stochastic differential equation $(2.1)$, where $b(x)=\left(b_{1}(x), \ldots, b_{n}(x)\right)$ is a Lipschitz vector field and

$$
\sigma(x)=\sum_{j=1}^{n}\left(1-x_{j}^{2}\right) e_{j} \otimes e_{j} \quad \forall x \in Q_{1} .
$$

Then, conditions (3.4), (3.5), and (3.6) hold true, and

$$
L_{0} \varphi=\frac{1}{2} \sum_{j=1}^{n}\left(1-x_{j}^{2}\right) \partial_{j}^{2} \varphi+\langle b(x), \nabla \varphi\rangle .
$$

Therefore, recalling (3.8), we conclude that $\stackrel{\circ}{Q}_{1}$ is invariant if and only if

$$
b_{j}(x) \frac{x_{j}}{\left|x_{j}\right|} \leq 0 \quad \forall x \in \partial Q_{1}, \forall j \in J(x) .
$$

Under the above assumption we have that semigroup $P_{t}$ has at most one invariant measure $\mu<<\mu_{2}$, whose existence is guaranteed if

$$
b_{j}(x) \frac{x_{j}}{\left|x_{j}\right|}<0 \quad \forall x \in \partial Q_{1}, \forall j \in J(x) .
$$


Example 5.10 Let us consider the stochastic differential equation in the closed unit ball $K=\bar{B}_{1} \subset \mathbb{R}^{n}$,

$$
\left\{\begin{array}{l}
d X(t)=b(X(t)) d t+\left(1-|X(t)|^{2}\right) d W(t), \quad t \geq 0 \\
X(0)=x
\end{array}\right.
$$

where $b: \bar{B}_{1} \rightarrow \mathbb{R}^{n}$ is a Lipschitz vector field. The corresponding Kolmogorov operator is

$$
L_{0} \varphi=\frac{1}{2}\left(1-|x|^{2}\right)^{2} \Delta \varphi+\langle b(x), \nabla \varphi\rangle .
$$

Applying Theorems 3.2 and 5.1, one checks easily that $B_{1}$ is invariant for $X$ if and only if

$$
\max _{|x|=1}\langle b(x), x\rangle \leq 0,
$$

and that $P_{t}$ has at most one invariant measure $\mu<<\mu_{2}$ under the above assumption. Moreover, by Theorem 5.7, such a measure does exist if

$$
\liminf _{|x| \uparrow 1} \frac{\langle b(x), x\rangle}{(1-|x|) \log (1-|x|)}>0 .
$$

Now, let us compute the density $\rho$ of $\mu$ with respect to Lebesgue's measure, in the case when $b(x)=\beta x$ (where $\beta$ is a given real number). Note that, for such a vector field,

$$
(5.12) \Longleftrightarrow \beta<0 \text {. }
$$

Differentiating both sides of equation (5.1) with respect to $t$, the problem reduces to finding an integrable function $\rho$ such that

$$
\operatorname{div}\left[\left(1-|x|^{2}\right)^{2} \nabla \rho(x)-2\left(1-|x|^{2}\right) \rho(x) x-2 \beta \rho(x) x\right]=0 \quad \forall x \in B_{1} .
$$

Therefore, it suffices to solve the equation

$$
\left(1-|x|^{2}\right)^{2} \nabla \rho(x)-2\left(1-|x|^{2}\right) \rho(x) x-2 \beta \rho(x) x=0 \quad \forall x \in B_{1},
$$

that is easily seen to possess the solution

$$
\rho(x)=\frac{1}{1-|x|^{2}} e^{\frac{\beta}{1-|x|^{2}}} \quad \forall x \in B_{1} .
$$

The above integrable function being integrable since $\beta<0$, (5.13) gives the required density. 


\section{Appendix}

We will prove Theorem 4.4 in three steps.

1. Existence and regularity. Observe that, since one can argue with the positive and negative part of $f$ separately, it suffices to prove the existence of a solution to (4.14) for $f \geq 0$. Having fixed $f$, define

$$
\varphi^{f}(x)=\int_{0}^{\infty} e^{-\lambda t} P_{t} f(x) d t \quad \forall x \in K .
$$

Then, as is well-known, $\varphi^{f} \in D(L)$ and

$$
L \varphi^{f}=\lambda \varphi^{f}-f \quad \text { in } K .
$$

Now, for $i \in \mathbb{N}$ large enough, let

$$
\varphi_{i}^{f}(x):=\int_{0}^{\infty} e^{-\lambda t} P_{t}^{i} f(x) d t \quad\left(x \in Q_{i}\right),
$$

where $P_{t}^{i}$ are the stopped semigroups defined in (4.6). Owing to (4.7),

$$
\varphi_{i}^{f}(x) \uparrow \varphi^{f}(x) \quad(i \rightarrow \infty) \quad \forall x \in \stackrel{\circ}{K} .
$$

Moreover, on account of (4.6),

$$
\varphi_{i}^{f}(x)=\int_{0}^{\infty} e^{-\lambda t} \mathbb{E}\left[f\left(X(t, x) \mathbb{1}_{t \leq \tau_{Q_{i}}(x)}\right] d t \quad \forall x \in Q_{i} .\right.
$$

Since our diffusion process is nondegenerate in $Q_{i}$ and $Q_{i}$ is a compact domain of class $C^{2,1}$, it is well-known that $\varphi_{i}^{f}$ satisfies

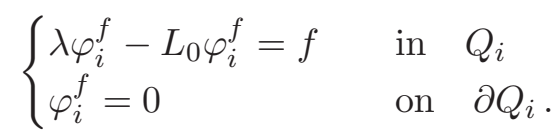

Thus, by classical elliptic theory we conclude that, for all $i \in \mathbb{N}, \varphi_{i}^{f}$ belongs to $H^{2}\left(Q_{i}\right)$. Also, for any open subset $A$ of $\stackrel{\circ}{K}$ such that $\bar{A} \subset \stackrel{\circ}{K}$,

$$
\left\|\varphi_{i}^{f}\right\|_{H^{2}(A)} \leq C_{A}
$$

for a suitable constant $C_{A}$, independent of $i$ (see, e.g., [23, Appendix A]). So, from (6.5) and (6.3) we deduce that $\varphi^{f} \in H_{\mathrm{loc}}^{2}(K)$, and (6.4) yields

$$
L_{0} \varphi^{f}=\lambda \varphi^{f}-f \quad \text { in } \quad \stackrel{\circ}{K} .
$$

Since the right-hand side above is continuous in $K,(6.6)$ holds on the closed domain $K$ and $L_{0} \varphi^{f} \in C(K)$. Therefore, $\varphi^{f} \in D\left(L_{0}\right)$ and, in view of (6.2), $L_{0} \varphi^{f}=L \varphi^{f}$. 
2. An auxilary problem. Let $\varphi^{1} \in D\left(L_{0}\right)$ be the solution of (4.14) for $f \equiv 1$, that we constructed in the previous step. Since $P_{t} 1=1$, by (6.1) we conclude that

$$
\varphi^{1}(x)=\frac{1}{\lambda} \quad \forall x \in K .
$$

Moreover, owing to (6.3),

$$
\varphi_{i}^{1}(x) \uparrow \frac{1}{\lambda} \quad(i \rightarrow \infty) \quad \forall x \in \stackrel{\circ}{K},
$$

where $\varphi_{i}^{1}$ is the solution of $(6.4)$ for $f \equiv 1$.

3. Uniqueness. We will show that, if

$$
\left\{\begin{array}{l}
u \in D\left(L_{0}\right) \\
\lambda u-L_{0} u=0 \quad \text { in } K,
\end{array}\right.
$$

then $u \equiv 0$. Let

$$
v(x):=\frac{1}{\lambda}-\frac{u(x)}{\lambda\left(1+\|u\|_{C(K)}\right)} \quad x \in K .
$$

Then

$$
\begin{cases}v \in D\left(L_{0}\right) & \\ v(x)>0 & \forall x \in K \\ \lambda v-L_{0} v=1 & \text { in } K .\end{cases}
$$

Therefore, comparing $v$ and the solution $\varphi_{i}^{1}$ of (6.4) for $f \equiv 1$ on $Q_{i}$, we obtain

$$
v(x) \geq \varphi_{i}^{1}(x) \quad \forall x \in Q_{i}
$$

for all $i \in \mathbb{N}$ large enough. Hence, in view of (6.7),

$$
v(x) \geq \frac{1}{\lambda} \quad \forall x \in K,
$$

which in turn implies that $u(x) \leq 0$ for all $x \in K$. By the same argument applied to $-u$ we conclude that $u \equiv 0$.

\section{Acknowledgement}

The authors are grateful to the anonymous referees for their constructive remarks, including useful bibliographical comments, which substantially improved the presentation of this paper. 


\section{References}

[1] AUBIN J.-P. and DA PRATO G., Stochastic viability and invariance, Annali Scuola Norm. Sup. Pisa (IV), 17, 595-613, 1990.

[2] AUBIN J.-P. and DA PRATO G, Stochastic Nagumo's viability theorem, Stochastic Anal. Appl., 13, 1-11, 1995.

[3] AUBIN J.-P. and DA PRATO G., The viability theorem for stochastic differential inclusion, Stochastic Anal. Appl., 16, 1-15, 1998.

[4] AUBIN J.-P. and FRANKOWSKA H., Set-Valued Analysis, Birkhäuser, Boston, 1990.

[5] BARDI M. and GOATIN P., Invariant sets for controlled degenerate diffusions: a viscosity solution approach, in "Stochastic analysis, control, optimization and application" (W.M.M. Mc Eneaney, G.G. Yin, Q. Zhang Eds.), Birkhäuser, 191-208, 1999.

[6] BARDI M. and JENSEN R., A geometric characterization of viable sets for controlled degenerate diffusions, Set-Valued Analysis, 10, 129-141, 2002 .

[7] BUCKDAHN R., PENG S., QUINCAMPOIX M. and RAINER C., Existence of stochastic control under state constraints, C. R. Acad. Sci. Paris, Série I, 327, 17-22, 1998.

[8] Cessenat M., DAUTRAY R., LEDANOIS G., LiONS P.-L., PARDOUX E., and SENTIS R., MÉthodes probabilistes pour LeS Équations DE LA PHYSique, Eyrolles, Paris, 1989.

[9] DA PRATO G., An introduction to infinite-dimensional analYsis, Springer-Verlag, Berlin, 2006.

[10] DA PRATO G. and FRANKOWSKA H., Stochastic viability for compact sets in terms of the distance function, Dynam. Systems Appl., 10, 177-184, 2001.

[11] DA PRATO G. and FRANKOWSKA H., Existence, uniqueness and regularity of the invariant measure for a class of elliptic degenerate operators, J. Diff. and Integral Eqs. Volume 17, 737-750, 2004.

[12] DA PRATO G. and FRANKOWSKA H., Invariance of stochastic control systems with deterministic arguments, J. Differential Equations, 200, $18-52,2004$. 
[13] DA PRATO G. and FRANKOWSKA H., Invariant measure for a class of parabolic degenerate equations, NoDEA, Volume 12, Number 4, 481$501,2005$.

[14] DA PRATO G. and FRANKOWSKA H., Stochastic viability of convex sets, J. Math. Anal. Appl. 333, no. 1, 151-163, 2007.

[15] DA PRATO G. and ZABCZYK J., ERgodicity For INFinite DimenSiOnAl Systems, London Mathematical Society Lecture Notes, n.229, Cambridge University, 1996.

[16] DELFOUR M.C. and ZOLESIO J.-P., Shape analysis via oriented distance function, J. Funct. Anal., Volume 123, 129-201, 1994.

[17] FRIEDMAN A. and PINSKY M.A. Asymptotic stability and spiraling properties of solutions of stochastic equations, Trans. Amer. Math. Soc. 186, 331-358, 1973.

[18] FRIEDman A., Stochastic differential Equations and AppliCAtions. Vol.1, Academic Presss, New York, 1975.

[19] FRIEDMAN A., Stochastic Differential Equations and APpliCAtions. Vol.2, Academic Presss, New York, 1976.

[20] FELLER W., On second order differential operators, Ann. of Math. (2) 61, 90-105, 1955.

[21] KATSOULAKIS M., Viscosity solutions of second order fully nonlinear elliptic equations with state constraints, Indiana Univ. Math. J. (2) 43, 493-519, 1994.

[22] LADYZHENSKAJA O.A, SOLONNIKOV V. A. and URAL'CEVA N.N., LinEAR AND QUASILINEAR EQUATIONS OF PARABOliC TYPE, Transl. Math. Monographs, Amer. Math. Soc., 1968.

[23] LORENZI L. and BERTOLDI M., AnAlytical Methods For Markov semigroups, Chapman \& Hill/CRC, 2006. 